\title{
Neumorraquis como diagnóstico diferencial asertivo para trauma de abdomen cerrado
}

\section{Neumorraquis as an assertive differential diagnosis for closed abdomen trauma}

\author{
Adán Araujo-López, * Alejandro Dimitri Hernández-García, * \\ Citlalli Berenice Rodríguez-Cervantes, ${ }^{\ddagger}$ Noemí Rodríguez-González, ${ }^{\S}$ \\ Jesús Olivares-Peraza, Miguel Martínez-Buendía, Óscar Sánchez-Martínez
}

\begin{abstract}
RESUMEN. Introducción: La neumatosis intervertebral es una entidad rara, en la cual el paciente no refiere sintomatología alguna. Ésta suele hallarse incidentalmente en los estudios de imagen, tanto en la resonancia magnética como en la tomografía computarizada; su localización casi siempre es en los tendones cervicales y, con frecuencia, se asocia con traumatismo, o bien con la enfermedad discal degenerativa coexistente. Caso clínico: Presentamos el caso de un hombre de 62 años, con trauma de abdomen cerrado por caída de tres metros, quien presentó datos de abdomen agudo, pero en la tomografía axial computarizada no presentó ninguna lesión evidente; su evolución fue adecuada, sólo presentó neumorraquis de T11-L2. Discusión: La presencia de aire en el canal medular es una situación poco frecuente. Es importante reconocer que no existe un tratamiento específico, debido a que se ha estudiado poco la enfermedad y porque el origen de la patología es diferente, el cual en la mayoría de los casos no sigue un mismo patrón; el tratamiento consta de antiinflamatorios, analgésicos y aporte de oxígeno suplementario para ayudar a barrer con el $\mathrm{CO}_{2}$ que está atrapado dentro de las cavidades vertebrales. Conclusión: La neumorraquis es una entidad rara que, si no se tiene en cuenta y no se analiza de forma completa a través de la tomografía axial computarizada, nunca se llegará al diagnóstico, por tanto, la proponemos como un diagnóstico diferencial que se debe de considerar, evitando laparotomías negativas.
\end{abstract}

Palabras clave: Neumorraquis, trauma de abdomen cerrado, diagnóstico.

ABSTRACT. Introduction: The intervertebral pneumatosis is a rare entity, the patient usually does not report any symptoms and is usually an incidental finding in the imaging studies in both magnetic resonance and computed tomography, its location is almost always in cervical tendons frequently associated with trauma or with coexisting degenerative disc disease. Case report: We present the case of a 62-year-old man with abdominal trauma due to a fall of 3 meters, presenting acute abdomen data, however in the CAT no obvious lesion, adequate evolution, only neumorraquis of T11-L2. Discussion: The presence of air in the medullary canal is a rare situation. It is important to recognize that there is no specific treatment, because the disease has been little studied and the origin of the pathology is different, in most cases it does not follow the same pattern, so that it consists of anti-inflammatories, analgesics, supplemental oxygen supply to help sweep the Co2 that is trapped inside the vertebral cavities. Conclusion: The neumorraquis is a rare entity, which if not taken

* Jefe de la División de Cirugía General.

‡ Cirujano General.

$\S$ Médico Interno de Pregrado de Cirugía General.

Hospital General de Cadereyta, Querétaro.

Recibido: 19 de Mayo de 2019.

Aceptado: 27 de Febrero de 2020.

\section{Correspondencia:}

Adan Araujo-López

Circuito Andamaxei No. 6, Col. Paseos del Bosque, CP 76910, Corregidora, Querétaro. Teléfono: 4427448305 Celular: 4444491203

E-mail: draraujolopez@gmail.com

Conflicto de Intereses: Todos los autores declaran que no existe ningún conflicto de intereses con respecto a la publicación de este artículo. 
into account, the Computed Axial Tomography is not analyzed completely, it will never reach the diagnosis, for which we propose it as a differential diagnosis that should be considered, avoiding negative laparotomies.

Keywords: Neumorraquis, abdominal blunt trauma, diagnosis.

\section{INTRODUCCIÓN}

El neumorraquis es una entidad rara que se ha relacionado de forma posterior con un traumatismo de abdomen o tórax. Esta entidad consiste en un atrapamiento de aire en el espacio epidural, subdural o subaracnoideo, dependiendo de la altura a la que esté el aire atrapado anatómicamente. De hecho, tiene varias etiologías, patologías y tratamientos, ${ }^{1}$ no obstante, es asintomático y se identifica como un hallazgo casual en los estudios radiológicos. ${ }^{2}$

Su diagnóstico suele ser sesgado por un traumatismo, y éste suele ser difícil, ya que por lo general el paciente no refiere sintomatología alguna y se halla incidentalmente en los estudios de imagen, tanto en la resonancia magnética como en la tomografía computarizada; su localización se encuentra en un $40 \%$ en vértebras cervicales, $30 \%$ en torácicas, $25 \%$ en lumbares y $5 \%$ en el sacro. ${ }^{3}$

\section{CASO CLÍNICO}

Presentamos el caso de un hombre de 62 años, cuyos antecedentes de importancia son: plastia inguinal derecha de 10 años e izquierda de hace ocho años (ambas con bloqueo subaracnoideo sin complicación referida por notas de anestesiología), catarata de ojo derecho e hipertensión arterial sistémica en tratamiento con $50 \mathrm{mg} /$ día de losartán.

Tres horas después de haber sufrido una caída de tres metros de altura y al caer sobre su costado izquierdo, ingresó a la sala de urgencia sin alteración del estado de conciencia. Fue traído por dolor abdominal y al momento de explorarlo, se encontró el abdomen blando, depresible, con peristalsis, sin signos de irritación peritoneal, pero en dermatomos de T11, T12 con alteración de sensibilidad y fuerza conservada.

Por ello, se realizó un ultrasonido FAST (que resultó negativo) y una tomografía axial computa- rizada abdominal por la evolución (Figuras 1 y 2), descartando alguna lesión de órgano sólido; el neumotórax sólo presentó aire en espacio epidural desde T11 a L2. Después, se inició manejo con oxígeno con $\mathrm{FiO}_{2}$ a 41\%, analgésicos opioides y antibióticos.

El paciente mejoró a las 48 horas; la tomografía axial computarizada de control mostró la persistencia de aire en el espacio epidural, abdomen sin lesiones ni liquido libre ni aire en cavidad peritoneal, por lo cual egresó con mejoría completa de su padecimiento.

\section{DISCUSIÓN}

Si bien la causa principal es el trauma, se han descrito otros motivos en la literatura, pudiéndose clasificar como traumáticos y no traumáticos..$^{4,5}$ Las causas que pueden motivar un neumorraquis son diversas: consumo de drogas, abscesos, ataques de asma o tos, vómitos

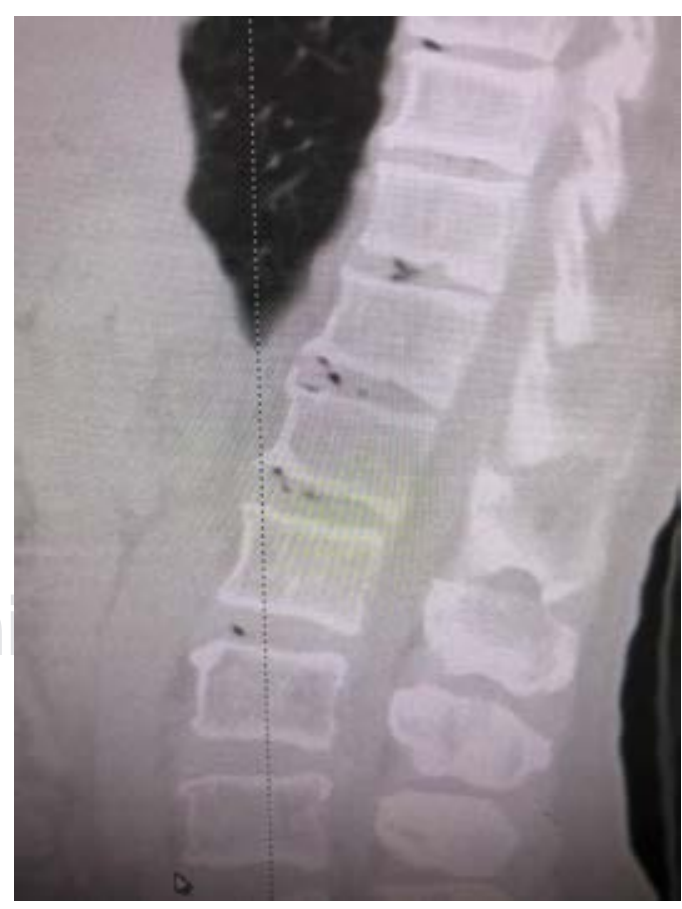

Figura 1: Corte sagital que muestra neumorraquis de T12-L1. 


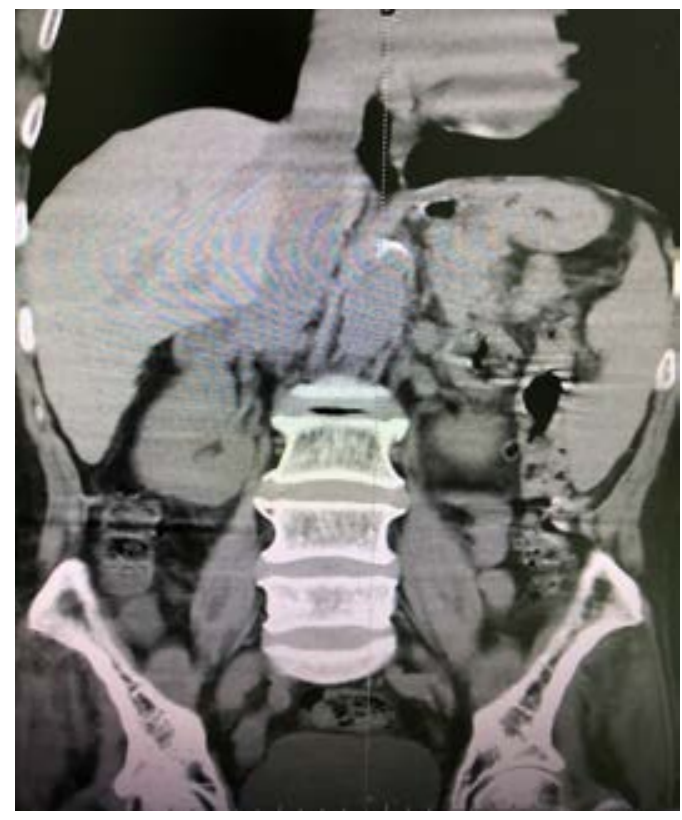

Figura 2: Corte coronal que muestra neumorraquis de T12-L1 sin lesiones orgánicas.

violentos, anestesia epidural, punción lumbar, cirugía o traumatismos de tórax, e intervención quirúrgica vertebral; más raramente se puede asociar con meningitis o neumoencéfalo. También esta patología es un indicador de lesión severa que, por lo general, clínicamente no se especifica dado su origen. ${ }^{5}$ Otra clasificación se puede describir como epidural (aire intraespinal o epidural) e intradural (aire intraespinal dentro del espacio subdural o subaracnoideo).
La diferenciación puede ser difícil en algunos casos. El diagnóstico generalmente se realiza por tomografía axial computarizada (en los cortes coronales), aunque las radiografías simples pueden detectar grandes volúmenes de aire intraespinal. ${ }^{6}$

Dentro del tratamiento es importante reconocer que no hay uno en específico, debido al poco estudio y conocimiento que se tiene de la enfermedad, así como por el origen de la misma. En la mayoría de los casos, no sigue un mismo patrón, por lo que su tratamiento consta de antiinflamatorios o analgésicos de predominio opioideo y aporte de oxígeno suplementario para barrer el $\mathrm{CO}_{2}$ atrapado en las cavidades vertebrales. ${ }^{7}$

\section{CONCLUSIÓN}

La presencia de aire en el canal medular es una situación poco frecuente, por lo que es importante reconocerla como un diagnóstico diferencial en aquellos pacientes que han sufrido un trauma de abdomen cerrado. Cabe mencionar que la exploración física, laboratorios e imagen descartan indicación de laparotomía exploradora.

Debido a que son enfermedades autolimitadas, el tratamiento dependerá de las condiciones que ocasionaron la enfermedad y comorbilidades del paciente. No obstante, se puede llegar a una patología grave promoviendo paresia parcial o total, por lo que, al tener en cuenta el diagnóstico, se pueden evitar complicaciones.

\section{BIBLIOGRAFÍA}

1. Kitagawa T, Fujiwara A, Tamai K, Kobayashi N, Saiki K, Omata $\mathrm{S}$ et al. Enlarging vertebral body pneumatocysts in the cervical spine. AJNR Am J Neuroradiol. 2004; 24 (8): 1707-1710.

2. Yamamoto T, Yoshiya S, Kurosaka M. Natural course of an intraosseous pneumatocyst of the cervical spine. AJR Am J Roentgenol. 2002; 22 (7): 1055-1961.

3. Abdrabou A. Vertebral pneumatocyst. Revista de RadioPedia. 2019; 10 (1): 102-105.

4. Salas SD. Caso 3-2015: masculino 19 años con neumorraquis post-traumático. Revista Clínica de la
Escuela de Medicina UCR-HSJD/Costa Rica. 2015; 5 (1): 1-3.

5. Newbold RG, Wiener MD, Vogler JB, Martinez S. Traumatic pneumorrhachis. American Roentgen Ray/EU. 2016; 7 (1): 201-208.

6. Goh BK, Yeo AW. Traumatic pneumorrhachis. J Trauma. 2005; 58 (8): 875-879.

7. Oertel M, Korinth M, Reinges M, Krings T, Terbeck S, Gilsbac J. Pathogenesis, diagnosis and management of pneumorrhachis. Eur Spine J. 2006; 15 (1): 636 643. 